\title{
Isotope effects on the pseudogap in high-temperature superconductors
} \author{
J. Karpinski ${ }^{3}$, H. Mutka ${ }^{4}$, K.A. Müller ${ }^{5}$ \\ ${ }^{1}$ Laboratory for Neutron Scattering, ETHZ \& PSI, 5232 Villigen PSI, Switzerland \\ ${ }^{2}$ Petersburg Nuclear Physics Insitute, Gatchina 188350, Russia \\ ${ }^{3}$ Laboratory for Solid State Physics, ETH Zürich, 8093 Zürich, Switzerland \\ ${ }^{4}$ Institute Laue-Langevin, B. P. 156, 38042 Grenoble Cedex, France \\ ${ }^{5}$ Physics Institute, University of Zürich, 8057 Zürich, Switzerland \\ Received: 18 July 2001/Accepted: 12 March 2002 - (C) Springer-Verlag 2002
}

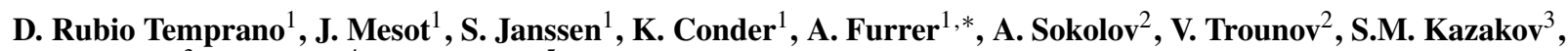

\begin{abstract}
Inelastic neutron scattering studies of the relaxation rate of crystal-field excitations have provided evidence for large oxygen and copper isotope effects on the pseudogap in high-temperature superconductors. Existing experiments in the slightly underdoped $\mathrm{HoBa}_{2} \mathrm{Cu}_{4} \mathrm{O}_{8}$ compound will be reviewed, as well as possible implications of such findings on the microscopic mechanism of high-temperature superconductivity. New relevant experiments performed in the $\mathrm{La}_{1-x} \mathrm{Sr}_{x} \mathrm{CuO}_{4}$ family will also be presented.
\end{abstract}

PACS: 74.72.Bk; 74.90.+n; 61.12.Ex

In the past few years, the underdoped region of hightemperature superconductors (HTSC) has attracted a great deal of attention due to the gradual development of a pseudogap at a characteristic temperature $T^{*}$, which lies significantly above $T_{\mathrm{c}}$ in the normal state. The pseudogap has a profound effect on a wide range of physical properties of the cuprates in the normal state. Although its exact nature and relation to superconductivity is still a subject of great controversy, it is widely believed that without the explanation of these unsual normal-state properties it is hard to expect a complete understanding of the microscopic mechanism of high-temperature superconductivity. This belief has been the motivation of considerable experimental work, and evidence for gap-like structures in the normal state has already been provided by a variety of experimental methods (see [1] for a recent review). However, the experimental situation regarding the doping dependence of the pseudogap is, up to some extent, contradictory. As a result of the experimental work two different generic phase diagrams are being debated [2]. In the first one, the pseudogap temperature $T^{*}$ falls from a high value at low doping to cross the superconducting region, going abruptly to zero at a doping level $p=0.19$ which is slightly above optimum doping. In this case the pseudogap is necessarily independent and competing with superconductivity and could implicate the existence of a quantum critical

*Corresponding author.

(Fax: +41-56/310-2939, E-mail: Albert.Furrer@psi.ch) point. In the second one, the slope of the $T^{*}$ line is somehow smaller in the underdoped regime and $T^{*}$ merges with the superconducting critical temperature in the overdoped side. This suggests that the superconducting gap and the pseudogap are closely related, and that some non-coherent sign (precursor pairing) of superconductivity persists in the normal state.

In order to contribute to the clarification of the present situation, we have performed neutron spectroscopy measurements for $\mathrm{HoBa}_{2} \mathrm{Cu}_{3} \mathrm{O}_{x}$ in the strongly underdoped $(x=6.46)$, the intermediately underdoped $(x=6.56)$ and the slightly overdoped $(x=6.96)$ regimes in order to establish a phase diagram for 123-type compounds [3]. The $\mathrm{HoBa}_{2} \mathrm{Cu}_{4} \mathrm{O}_{8}$ compound was also included, since it is equivalent to 123 doping of $x \approx 6.78$. The measurements gave evidence for the presence of a pseudogap above the superconducting temperature for all the doping levels considered, even in the slightly overdoped regime. The data also suggest a linear decrease of $T^{*}$ with increasing doping. This result supports the second of the phase diagrams mentioned above, as well as the existence of a precursor pairing above $T_{\mathrm{c}}$. Isotope effects may provide important information about the pairing mechanism, as they were also crucial to establish the BCS model for conventional superconductors. If the local pairing occurs at the pseudogap opening, isotope effects may be somehow bigger at $T^{*}$ than at $T_{\mathrm{c}}$, where the phase coherence sets in.

In the following, existing experiments searching for isotope effects on the pseudogap temperature are reviewed. Moreover, relevant new measurements in the $\mathrm{La}_{2-x} \mathrm{Sr}_{x} \mathrm{CuO}_{4}$ family are also presented.

\section{Crystal-field relaxation}

The principle of neutron spectroscopic investigations of the crystal-field interaction in rare-earth based high- $T_{\mathrm{c}}$ superconductors was described in a review article [4]. By this technique transitions between different crystal-field levels associated with the rare-earth ions can be directly measured. In the normal metallic state the excited crystal-field levels interact with phonons, spin fluctuations, and charge carriers (electrons 
or holes), which limit the lifetime of the excitation, thus the observed crystal-field transitions exhibit line broadening. The relaxation with the charge carriers is the dominant relaxation mechanism. Therefore we describe the linewidth in the normal state by the modified Korringa law [5]

$$
\begin{aligned}
\Gamma_{n}^{i j}(T)= & 2 J_{\mathrm{ex}}^{2}\left[M_{i j}^{2} \operatorname{coth}\left(\frac{\hbar \omega_{i j}}{2 k_{\mathrm{B}} T}\right) \chi^{\prime \prime}\left(\hbar \omega_{i j}\right)\right. \\
& +\sum_{n \neq i} M_{\mathrm{in}}^{2} \frac{\chi^{\prime \prime}\left(\hbar \omega_{\mathrm{in}}\right)}{\exp \left(\frac{\hbar \omega_{\mathrm{in}}}{k_{\mathrm{B}} T}\right)-1} \\
& \left.+\sum_{n \neq j} M_{n j}^{2} \frac{\chi^{\prime \prime}\left(\hbar \omega_{n j}\right)}{\exp \left(\frac{\hbar \omega_{n j}}{k_{\mathrm{B}} T}\right)-1}\right]
\end{aligned}
$$

where $J_{\text {ex }}$ is the exchange integral between the $4 f$ electrons of the rare-earth ions and the charge carriers, $M_{i j}$ the matrix element of the crystal-field transition $|i\rangle \rightarrow|j\rangle$ with energy transfer $\hbar \omega_{i j}=\hbar \omega_{i}-\hbar \omega_{j}$, and the factor $\chi^{\prime \prime}\left(\hbar \omega_{i j}\right)=$ $\pi N^{2}\left(E_{\mathrm{F}}\right) \hbar \omega_{i j}$ is the generalised susceptibility of the copperoxide layers in the normal state with $N\left(E_{\mathrm{F}}\right)$ being the densityof-states of the charge carriers at the Fermi energy $E_{\mathrm{F}}$. In superconducting compounds, however, the pairing of the charge carriers creates an energy gap $\Delta$ below the superconducting transition temperature $T_{\mathrm{c}}$ and thereby suppresses $N\left(E_{\mathrm{F}}\right)$, thus crystal-field excitations with energy $\hbar \omega_{i j}<2 \Delta$ do not have enough energy to span the gap, and consequently there is no interaction with the charge carriers. For an isotropic gap function the intrinsic linewidth in the superconducting state is then given by

$$
\Gamma_{\mathrm{s}}(T)=\Gamma_{\mathrm{n}}(T) \exp \left(-\frac{\Delta}{k_{\mathrm{B}} T}\right)
$$

This means that $\Gamma_{\mathrm{s}}\left(T<<T_{\mathrm{c}}\right) \approx 0$, and line broadening sets in just below $T_{\mathrm{c}}$ where the superconducting gap opens. The exponential temperature dependence of $\Gamma_{\mathrm{s}}(T)$ was demonstrated in a neutron spectroscopic study on the classical superconductor $\mathrm{La}_{1-x} \mathrm{~Tb}_{x} \mathrm{Al}_{2}$ [6] as well as for the high- $T_{\mathrm{c}}$ compound $\mathrm{HoBa}_{2} \mathrm{Cu}_{3} \mathrm{O}_{6.96}$ [3]. On the other hand, neutron experiments performed for $\mathrm{Ho}_{0.1} \mathrm{Y}_{0.9} \mathrm{Ba}_{2} \mathrm{Cu}_{3} \mathrm{O}_{7}$ [5] revealed an unusual temperature dependence, i.e., $\Gamma_{\mathrm{s}}(T)$ does not follow the behavior predicted by (2), but increases already far below $T_{\mathrm{c}}$. This was ascribed to a high degree of gap anisotropy, since an anisotropic gap function gives rise to certain relaxation channels even at $T \ll T_{\mathrm{c}}$, particularly along the directions involving nodes. An anisotropic gap function was also inferred from neutron crystal-field studies on the high- $T_{\mathrm{c}}$ compounds $\mathrm{HoBa}_{2} \mathrm{Cu}_{4} \mathrm{O}_{8}$ and $\mathrm{Er}_{2} \mathrm{Ba}_{4} \mathrm{Cu}_{7} \mathrm{O}_{15}$ [7].

\section{The oxygen and copper isotope effects on $\mathrm{HoBa}_{2} \mathrm{Cu}_{4} \mathrm{O}_{8}$}

We have performed inelastic neutron scattering experiments to study the isotope effect on the relaxation rate of crystalfield excitations in $\mathrm{HoBa}_{2} \mathrm{Cu}_{4} \mathrm{O}_{8}$ by substituting ${ }^{16} \mathrm{O}$ vs. ${ }^{18} \mathrm{O}$. Details about sample preparation can be found in [8]. Neutron diffraction experiments confirmed the single-phase character of the samples. The critical temperatures $T_{\mathrm{c}}$ of the ${ }^{16} \mathrm{O}$ and ${ }^{18} \mathrm{O}$ compounds were determined by magnetometry to be $79 \mathrm{~K}$ and $78.5 \mathrm{~K}$, respectively. The observed oxygen isotope shift on $T_{\mathrm{c}}$, i.e., $\Delta T_{\mathrm{c}} \approx-0.5 \mathrm{~K}$ can be described by the isotope coefficient $\alpha$ defined by the relation $T_{\mathrm{c}} \propto M^{-\alpha}$, where $M$ is the mass of the oxygen ion. For the above compounds we derive $\alpha=0.05 \pm 0.02$, in agreement with literature data [9, 10].

The neutron scattering experiments were performed on the high resolution time-of-flight spectrometers IN5 and FOCUS installed at the Institute Laue-Langevin (Grenoble) and at the Paul Scherrer Institute (Villigen), respectively. In both experiments the incident neutron wavelength was $5 \AA$, giving an energy resolution of $90 \mu \mathrm{eV}$ at the elastic position. The raw data were corrected for absorption, detector efficiency, background and detailed balance effects according to standard procedures.

Figure 1 shows energy spectra for $\mathrm{HoBa}_{2} \mathrm{Cu}_{4}{ }^{18} \mathrm{O}_{8}$. There are two strong ground-state crystal-field transitions at $\hbar \omega_{1}=$ $0.6 \mathrm{meV}$ and $\hbar \omega_{2}=1.3 \mathrm{meV}$ [11], i.e., $\hbar \omega_{i} \ll 2 \Delta_{\max }$ $(\sim 66 \mathrm{meV}[7])$. With increasing temperature the crystalfield transitions exhibit line broadening. In addition, excited crystal-field states become increasingly populated giving rise to excited crystal-field transitions. The energy spectra were fitted according to the neutron cross-section for crystal-field transitions which is given in the dipole approximation by

$$
\frac{d^{2} \sigma}{\mathrm{d} \Omega \mathrm{d} \omega} \propto \sum_{i, j} M_{i j}^{2} \exp \left(-\frac{\hbar \omega_{i}}{k_{\mathrm{B}} T}\right) / Z \delta\left(\hbar \omega_{i j} \pm \hbar \omega\right)
$$

where $Z$ is the partition function, and $\hbar \omega_{i j}=\hbar \omega_{j}-\hbar \omega_{i} . M_{i j}$ and $\hbar \omega_{i j}$ were kept fixed at the values obtained for the ${ }^{16} \mathrm{O}$ compound [7]. The final spectra were obtained by convoluting the instrumental resolution function with the crystal-field intrinsic Lorentzian function, whose linewidth is given in the normal state by the modified Korringa law. (1) was also used to correlate the relaxation widths of the different crystal-field transitions, so that the only free parameters in the fitting procedure were then an overall scale factor for the intensities and a temperature-dependent linewidth $\Gamma(T) \sim\left[J_{\mathrm{ex}} N\left(E_{\mathrm{F}}\right)\right]^{2}$. The results of the fitting procedure are shown by solid lines in Fig. 1.

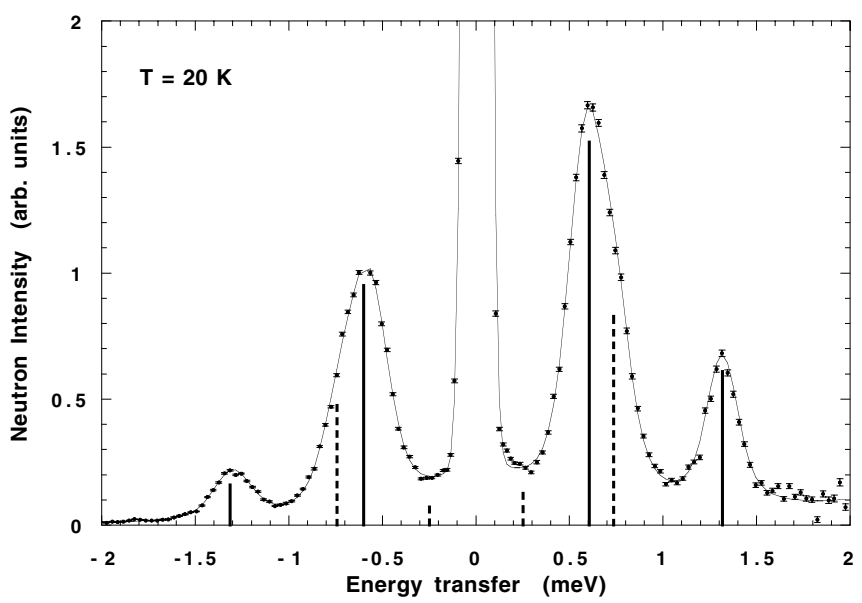

Fig. 1. Energy spectra of the neutrons scattered from $\mathrm{HoBa}_{2} \mathrm{Cu}_{4}{ }^{18} \mathrm{O}_{8}$ as taken on IN5. The solid lines are fits to the data which include the relaxation as described in the text. The solid and dashed vertical bars indicate the energy and intensity of the ground-state and excited-state crystal-field transitions, respectively, in the low-energy part of the spectra 
Figure 2 (upper panel) shows the temperature dependence of the intrinsic linewidth (HWHM) corresponding to the $\Gamma_{3}^{(1)} \rightarrow \Gamma_{4}^{(1)}$ transition from the ground state to the first excited state at $\hbar \omega=0.6 \mathrm{meV}$, derived for the ${ }^{18} \mathrm{O}$ compound. The two data sets (IN5 and FOCUS) are rather consistent. The linewidth is zero below $60 \mathrm{~K}$, then it increases almost linearly up to $210 \mathrm{~K}$. The linewidth is step-like enhanced between 210 and $220 \mathrm{~K}$. Above $220 \mathrm{~K}$ it increases again linearly as expected for the normal state. We therefore identify the temperature where the step-like enhancement occurs with the temperature where the pseudogap opens, i.e., we set $T^{*} \approx 220 \mathrm{~K}$. From the high-temperature data $(T \geq 220 \mathrm{~K})$ we obtained $J_{\mathrm{ex}} N\left(E_{\mathrm{F}}\right)=0.0063 \pm 0.0007$. Figure 2 (lower panel) shows the temperature dependence of the intrinsic linewidth derived for the same crystal-field transition in the ${ }^{16} \mathrm{O}$ compound. The figure includes earlier data taken on IN5 [7] as well as the data obtained on FOCUS for the back-exchanged compound. The nice consistency of the two data sets confirms the stability of the "1248" structure against oxygen isotope substitution. We recognise that the relaxation behavior of the ${ }^{16} \mathrm{O}$ sample has a similar shape as for the isotope substituted ${ }^{18} \mathrm{O}$ compound, but the step-like enhancement of the linewidth occurs at much lower temperatures between 160 and $170 \mathrm{~K}$, i.e., $T^{*} \approx 170 \mathrm{~K}$ [7]. From the high-temperature data $(T \geq 170 \mathrm{~K})$ we derived $J_{\mathrm{ex}} N\left(E_{\mathrm{F}}\right)=0.0060 \pm 0.0007$ which agrees within

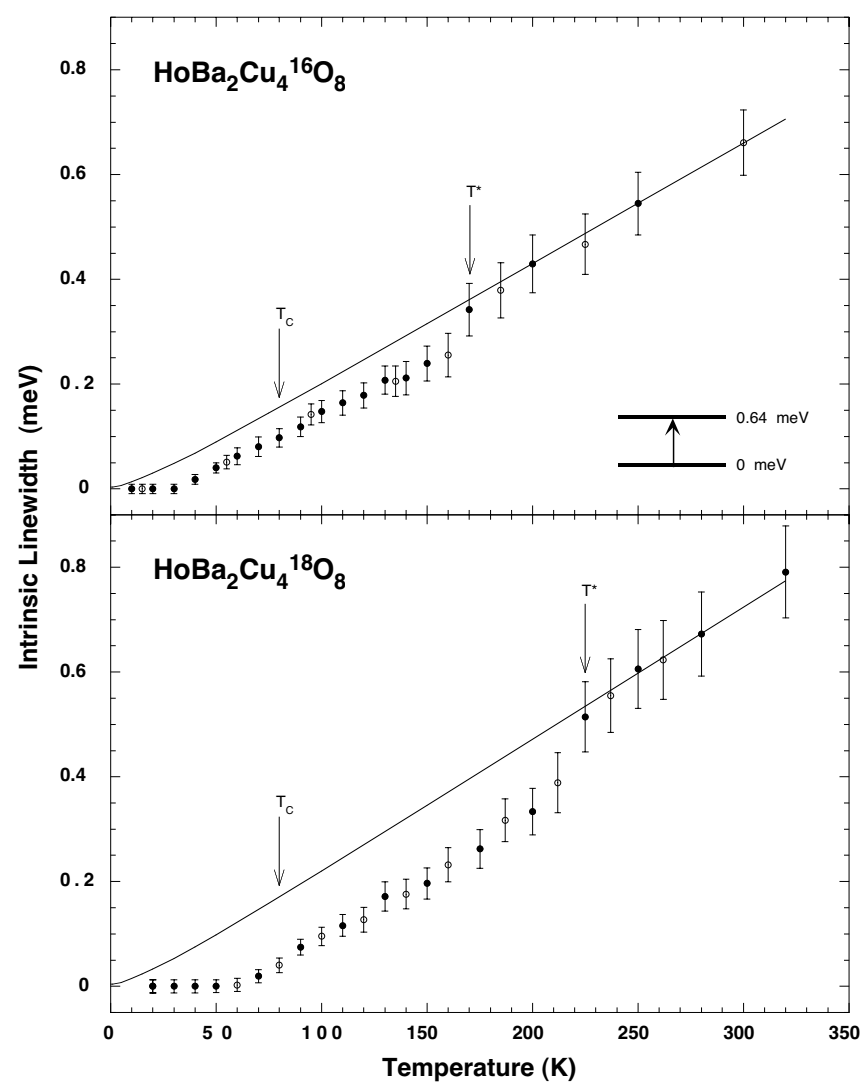

Fig. 2. Temperature dependence of the intrinsic linewidth $\Gamma(T)$ (HWHM) corresponding to the $\Gamma_{3}^{(1)} \rightarrow \Gamma_{4}^{(1)}$ ground state transition in $\mathrm{HoBa}_{2} \mathrm{Cu}_{4}{ }^{18} \mathrm{O}_{8}$ and $\mathrm{HoBa}_{2} \mathrm{Cu}_{4}{ }^{16} \mathrm{O}_{8}$. The lines denote the linewidth in the normal state calculated from (1). The full and open circles correspond to the data taken on IN5 and FOCUS, respectively. The FOCUS data for $\mathrm{HoBa}_{2} \mathrm{Cu}_{4}{ }^{16} \mathrm{O}_{8}$ refer to the back-exchanged compound the experimental error with the value determined for the ${ }^{18} \mathrm{O}$ compound. Our experiments give therefore evidence for a large isotope shift $\Delta T^{*} \approx 50 \mathrm{~K}$ which, in analogy to the isotope effect on $T_{\mathrm{c}}$, gives rise to an isotope coefficient $\alpha_{\mathrm{O}}^{*} \approx-2.2[8]$.

This result is in complete disagreement with the results of NMR and NQR experiments [9,10], which resulted in either an absence or a very small isotope shift $\Delta T^{*}$. All these methods are local probes with an interaction range of a few $\AA$. The major difference, however, is the time over which the spectral response is collected. NMR and NQR techniques work at $10-100 \mathrm{MHz}$ frequencies, thus they are insensitive to any mechanisms which are faster than about $10^{-8} \mathrm{~s}$. Neutron spectroscopy involves energies of some $\mathrm{meV}$ and is therefore susceptible to dynamical effects of the order of $10^{-13} \mathrm{~s}$. The time scale associated with each experimental method is essential for the detection of dynamical correlations. E.g., the time scales for spin and lattice fluctuations are of the order of $10^{-12} \mathrm{~s}$, thus they can easily be resolved by neutron spectroscopy, but not by NMR and NQR experiments. We can therefore immediately conclude that the mechanism giving rise to the isotope effect on the pseudogap has to involve a time scale $10^{-8} \mathrm{~s}>>\tau>10^{-13} \mathrm{~s}$. The isotope effect on $T^{*}$ measured by neutron crystal-field spectroscopy is strikingly similar to X-ray near-edge absorption spectroscopy (XANES) experiments, which revealed a huge isotope effect associated with the onset of local lattice fluctuations in $\mathrm{La}_{2-x} \mathrm{Sr}_{x} \mathrm{CuO}_{4}(x=0.06)$, whose characteristic temperature $T^{\prime}$ increases from 110 to $170 \mathrm{~K}$ upon replacing ${ }^{16} \mathrm{O}$ by ${ }^{18} \mathrm{O}$ [12]. XANES is an even faster technique with a time scale of $10^{-15} \mathrm{~s}$. The oxygen isotope effect on $T^{*}$ is actually expected from the polaron-like mechanism associated with the Jahn-Teller phonon modes. The width of the polaronic band $W$ depends on the ionic mass $M$ according to:

$W \propto e^{-\gamma \sqrt{M}}$

where $\gamma$ is a dimensionless parameter with $0<\gamma<1$ [13]. An increase in the ionic mass results in a narrowing of the polaronic band, so the separation $\Delta$ with respect to the $\mathrm{O}_{2 p}$ band increases and, therefore, $T^{*}$ also increases. Several theoretical attempts have been made in order to incorporate this finding into a model which describes the interaction between the charge carriers and the relevant phonon modes $[14,15]$. However, as shown below, not only the oxygen phonon modes have to be considered.

More relevant information can be obtained by studying isotope effects on $T^{*}$ with respect to other elements which are also directly involved in the superconducting mechanism, like copper. Recently, we performed a neutron crystalfield spectroscopy study of the copper isotope effect on $T^{*}$ in $\mathrm{HoBa}_{2} \mathrm{Cu}_{4} \mathrm{O}_{8}$. Details about sample preparation can be found in [16]. The critical temperatures of the ${ }^{63} \mathrm{Cu}$ and ${ }^{65} \mathrm{Cu}$ compounds were measured by magnetometry to be $79 \mathrm{~K}$ and $78.6 \mathrm{~K}$, respectively, giving a copper isotope shift $\Delta T_{\mathrm{c}}=$ $-0.4 \mathrm{~K}$. The neutron spectroscopic experiments were performed on FOCUS at SINQ, with an incident neutron wavelength of $4.8 \AA\left(\Gamma_{\mathrm{E}} \approx 85 \mu \mathrm{eV}\right)$.

The linewidth behaviour is qualitatively the same as that shown in the Fig. 2. However, the opening of the pseudogap takes place at $T^{*} \approx 160$ and $185 \mathrm{~K}$ for the $\mathrm{HoBa}_{2}{ }^{6} \mathrm{Cu}_{4} \mathrm{O}_{8}$ and $\mathrm{HoBa}_{2}{ }^{65} \mathrm{Cu}_{4} \mathrm{O}_{8}$ compounds, respectively [16]. These experi- 
ments give evidence therefore for a large copper isotope effect on the pseudogap, i.e. $\Delta T^{*} \approx 25 \mathrm{~K}\left(\alpha_{\mathrm{Cu}}^{*} \approx-4.9\right)$ [16].

The relevant mechanism giving rise to a copper isotope effect on $T^{*}$ has to be associated with a local copper phonon mode. This could be the umbrella-type motion, which involves out plane displacements of the copper ions [17]. The present results strongly suggest that the appropriate copper phonon modes have to be included in the corresponding hamiltonian describing the interaction between the charge carriers and the relevant phonon modes.

\section{The copper isotope effect on $\mathrm{La}_{1.81} \mathrm{Ho}_{0.04} \mathrm{Sr}_{0.15} \mathrm{CuO}_{4}$}

In order to shed light on the copper isotope effect on the pseudogap in $\mathrm{HoBa}_{2} \mathrm{Cu}_{4} \mathrm{O}_{8}$, we have performed new experiments on the $\mathrm{La}_{2-x} \mathrm{Sr}_{x} \mathrm{CuO}_{4}$ family, because this perovskite structure presents neither chains nor buckling in the $\mathrm{CuO}_{2}$ planes. Therefore, the umbrella-type motion is not present and cannot be responsible for a copper isotope effect, if there is any.

Details about sample preparation will be given elsewhere. Neutron powder diffraction confirmed the single-phase character of the samples. The critical temperatures of the ${ }^{63} \mathrm{Cu}$ and ${ }^{65} \mathrm{Cu}$ compounds were measured by magnetometry to be $32.2 \pm 0.2 \mathrm{~K}$ and $32.2 \pm 0.2 \mathrm{~K}$, respectively, giving no copper isotope shift within the experimental uncertainties.

The inelastic neutron scattering experiments were performed on FOCUS at SINQ. The incident neutron wavelength was $5.75 \AA\left(\Gamma_{\mathrm{E}} \approx 45 \mu \mathrm{eV}\right)$.

Figure 3 shows low-energy spectra of neutrons scattered from $\mathrm{La}_{1.81} \mathrm{Ho}_{0.04} \mathrm{Sr}_{0.15}{ }^{63} \mathrm{CuO}_{4}$. With increasing temperature the only crystal-field transition present, at $\hbar \omega \approx 0.18 \mathrm{meV}$, exhibits line broadening. The solid line represents a profile fit to the data as explained in Sect. 3. The crystalfield spectra for the copper isotope substituted compound $\mathrm{La}_{1.81} \mathrm{Ho}_{0.04} \mathrm{Sr}_{0.15}{ }^{65} \mathrm{CuO}_{4}$ look qualitatively the same.

Figure 4 displays the temperature dependence of the intrinsic linewidth (FWHM) corresponding to the $\Gamma_{1}^{(1)} \rightarrow \Gamma_{3}^{(1)}$ transition, derived for $\mathrm{La}_{1.81} \mathrm{Ho}_{0.04} \mathrm{Sr}_{0.15}{ }^{63} \mathrm{CuO}_{4}$ (upper pannel) and for $\mathrm{La}_{1.81} \mathrm{Ho}_{0.04} \mathrm{Sr}_{0.15}{ }^{65} \mathrm{CuO}_{4}$ (lower pannel). The linewidth is constant at the lowest temperatures, being the

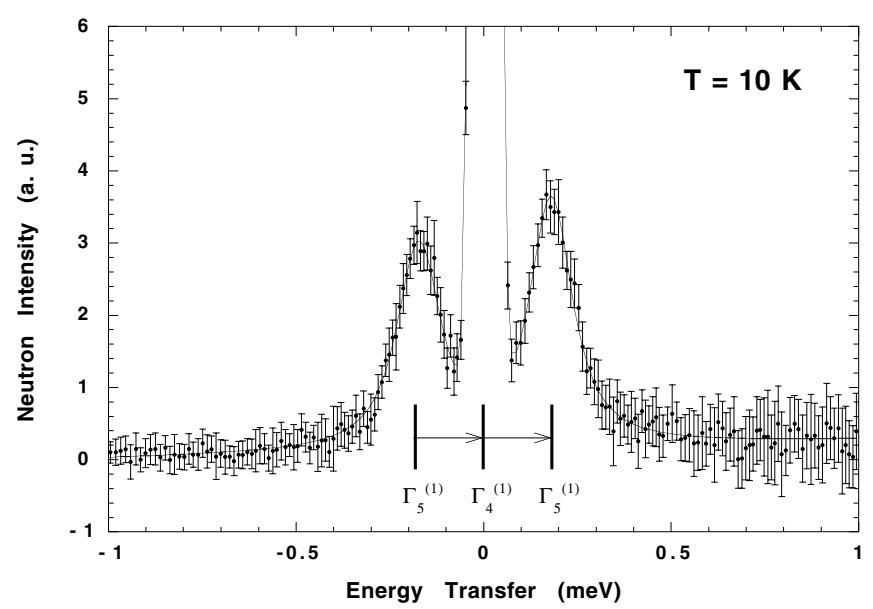

Fig. 3. Energy spectra of the neutrons scattered from $\mathrm{La}_{1.81} \mathrm{Ho}_{0.04} \mathrm{Sr}_{0.15}^{63}$. $\mathrm{CuO}_{4}$ as taken on FOCUS. The solid lines are profile fits to the data

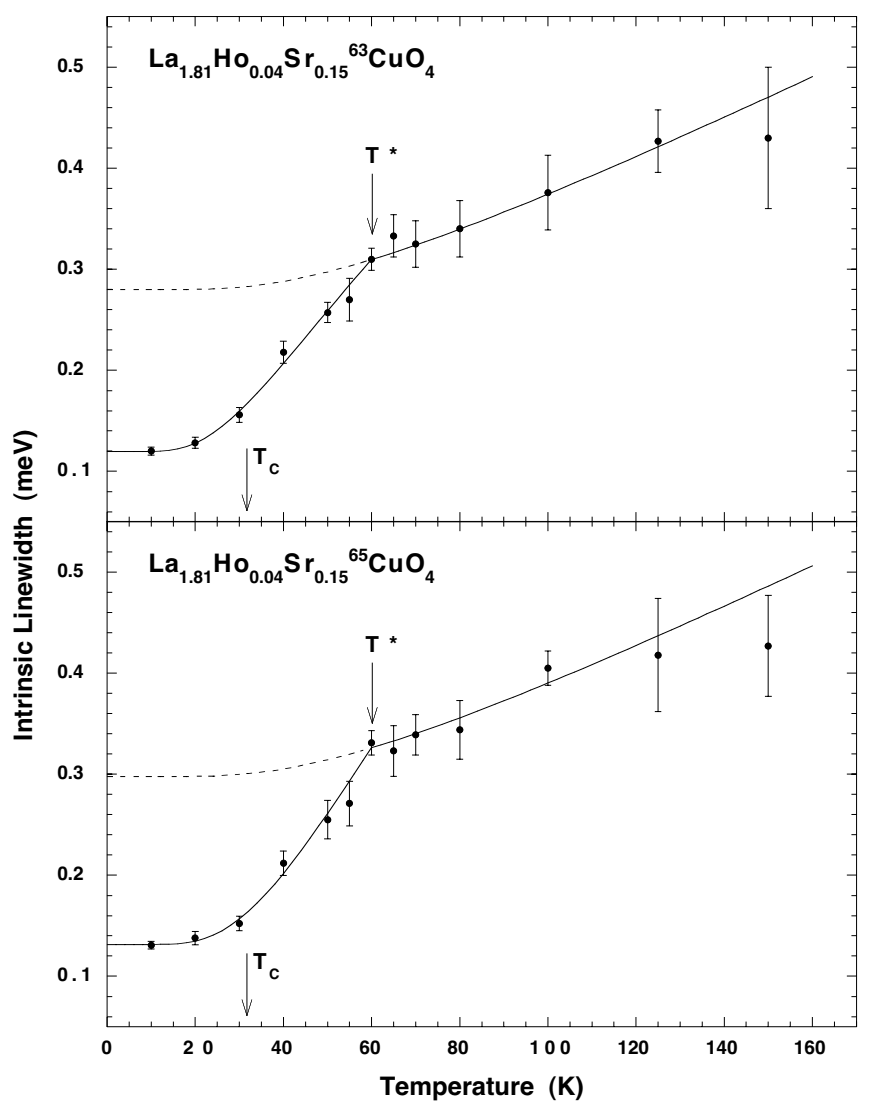

Fig. 4. Temperature dependence of the intrinsic linewidth $\Gamma(T)$ (FWHM) corresponding to the $\Gamma_{1}^{(1)} \rightarrow \Gamma_{3}^{(1)}$ ground state transition in $\mathrm{La}_{1.81} \mathrm{Ho}_{0.04}$. $\mathrm{Sr}_{0.15}{ }^{63} \mathrm{CuO}_{4}$ and $\mathrm{La}_{1.81} \mathrm{Ho}_{0.04} \mathrm{Sr}_{0.15}{ }^{65} \mathrm{CuO}_{4}$. The solid lines denote the linewidth behaviour in the superconducting and in the nornal state calculated from (2) and (1), respectively

residual value of about $0.1 \mathrm{meV}$ due to the chemical disorder around the $\mathrm{Ho}^{+3}$ ions. With increasing temperature the linewidth raises rapidly up to $60 \mathrm{~K}$, and from there on the increase is much slower and can be perfectly described by the Korringa law. We therefore set $T^{*} \approx 60 \mathrm{~K}$ for both compounds, i.e. there is no evidence for a copper isotope effect on the pseudogap in $\mathrm{La}_{1.81} \mathrm{Ho}_{0.04} \mathrm{Sr}_{0.15} \mathrm{CuO}_{4}$. This is in strong contrast to $\mathrm{HoBa}_{2} \mathrm{Cu}_{4} \mathrm{O}_{8}$ (see Sect. 3) and supports the idea that the umbrella-type mode may be responsible for the copper isotope effect on the pseudogap found for $\mathrm{HoBa}_{2} \mathrm{Cu}_{4} \mathrm{O}_{8}$.

\section{Conclusion}

The inelastic neutron scattering experiments presented provide evidence for the existence of large oxygen and copper isotope effects on the pseudogap temperature $T^{*}$ in $\mathrm{HoBa}_{2} \mathrm{Cu}_{4} \mathrm{O}_{8}$. This is an indication that phonons or lattice fluctuations are involved in the pairing mechanism. The oxygen isotope effect can be explained by the polaron-like mechanism associated with the Jahn-Teller phonon modes, whereas for the copper case it has to be associated with a local copper phonon mode. By comparison with new experiments on the $\mathrm{La}_{2-x} \mathrm{Sr}_{x} \mathrm{CuO}_{4}$ family, which provide no copper isotope effect for $T^{*}$, it is possible to assign this local mode to the umbrella-type motion. Of special importance are also the measurements on the $\mathrm{HoBa}_{2} \mathrm{Cu}_{3} \mathrm{O}_{x}$ compounds [3], 
which clearly show a pseudogap opening at $T^{*}>T_{\mathrm{c}}$ for all the doping levels considered, even in the slightly overdoped regime.

\section{References}

1. T. Timusk, B. Statt: Rep. Prog. Phys. 62, 61 (1999)

2. J.L. Tallon, J.W. Loram: Physica C 349, 53 (2001)

3. D. Rubio, J. Mesot, K. Conder, S. Janssen, H. Mutka, A. Furrer: J. Supercond. 13, 727 (2000)

4. J. Mesot, A. Furrer: J. Supercond. 10, 623 (1997)

5. A.T. Boothroyd, A. Mukherjee, A.P. Murani: Phys. Rev. Lett. 77, 1600 (1996)

6. R. Feile, M. Loewenhaupt, J.K. Kjems, H.E. Hoenig: Phys. Rev. Lett. 47, 610 (1981)

7. J. Mesot, G. Böttger, H. Mutka, A. Furrer: Europhys. Lett. 44, 498 (1998)
8. D. Rubio Temprano, J. Mesot, S. Janssen, K. Conder, A. Furrer H. Mutka, K.A. Müller: Phys. Rev. Lett. 84, 1990 (2000)

9. G.V.M. Williams, J.L. Tallon, J.W. Quilty, H.J. Trodahl, N.E. Flower: Phys. Rev. Lett. 80, 377 (1998)

10. F. Raffa, T. Ohno, M. Mali, J. Roos, D. Brinkmann, K. Conder M. Eremin: Phys. Rev. Lett. 81, 5912 (1998)

11. B. Roessli, P. Fischer, M. Guillaume, J. Mesot, U. Staub, M. Zolliker, A. Furrer, E. Kaldis, J. Karpinski, E. Jilek: J. Phys.: Cond. Mat. 6, 4147 (1994)

12. A. Lanzara, G.M. Zhao, N.L. Saini, A. Bianconi, K. Conder, H. Keller, K.A. Müller: J. Phys.: Cond. Mat. 11, L541 (1999)

13. M. Medarde, P. Lacorre, K. Conder, F. Fauth, A. Furrer: Phys. Rev. Lett. 80, 2397 (1998)

14. L.P. Gor'kov: J. Supercond. 13, 765 (2000)

15. A. Bussmann-Holder: J. Supercond. 13, 773 (2000)

16. D. Rubio Temprano, J. Mesot, S. Janssen, K. Conder, A. Furrer, A. Sokolov, V. Trounov, S.M. Kazakov, J. Karpinski, K.A. Müller: Eur. Phys. J. B, Rapid Note 19, 5 (2001)

17. J. Röhler: Physica C 341-348, 2151 (2000) 